\title{
The Neural Pathways for the Hypothetic Nociceptive-Sympathetic Coupling of NBA for Pain Sensation
}

\author{
Zi-Jian Cai \\ CaiFortune Consulting, Suzhou, China \\ Email:hrsh8@126.com
}

How to cite this paper: Cai, Z.-J. (2019) The Neural Pathways for the Hypothetic Nociceptive-Sympathetic Coupling of NBA for Pain Sensation. Open Access Library Journal, 6: e5780.

https://doi.org/10.4236/oalib.1105780

Received: September 11, 2019

Accepted: October 5, 2019

Published: October 8, 2019

Copyright $\odot 2019$ by author(s) and Open Access Library Inc.

This work is licensed under the Creative Commons Attribution International License (CC BY 4.0).

http://creativecommons.org/licenses/by/4.0/

\begin{abstract}
In May 2016, it was suggested by the US athletes playing games of National Basketball Association (NBA) in television that the pain sensation might result from the post-sensory nociceptive-sympathetic coupling. In this review, it is attempted to newly delineate the underlying neural pathways for this post-sensory nociceptive-sympathetic coupling. Based on the contemporary feedback neural circuits of pain transmission in spinal cord, it is summarized that the $\mathrm{A} \delta$ - and $\mathrm{C}$-fibers relay the sensory noxious signals to the laminae I, II and $\mathrm{V}$ of dorsal spinal horn, and in turn activate the periaqueductal grey (PAG) in midbrain, which feedbacks via raphe to inhibit the nociceptive transmission of spinal cord. Herein it is newly added that, as extension of the contemporary feedback pain circuits, the neurons in laminae I and V of dorsal spinal horn as well as those in PAG can additionally activate the sympathetic outputs, completing the post-sensory nociceptive-sympathetic coupling. It is emphasized that such nociceptive-sympathetic coupling would contribute at least partially to the pain sensation because of sympathetically maintained pain. It is also pointed out that the neurons in parabrachial area (PB) can concurrently relay the nociceptive inputs from spinal cord while regulating the respiration, coordinating the nociceptive and respiratory activities during pain sensation. Because both PAG and PB are below thalamus, it is speculated that these sympathetic and respiratory associations with pain are primitive and universal in evolution in vertebrates. It is expected to promote this hypothesis to revise the pain mechanisms in vertebrates and the relevant investigations in the future.
\end{abstract}

\section{Subject Areas}

Anaesthesiology \& Pain Management, Anatomy \& Physiology, Neuroscience 


\section{Keywords}

Pain, A $\delta$-Fiber, C-Fiber, Spinal Nociceptive-Sympathetic Coupling,

Periaqueductal Grey, Sympathetically Maintained Pain

\section{Introduction}

On May 6, 2016, the US athletes playing games of National Basketball Association (NBA) suggested in television that the sensation of pain might result necessarily from the mechanism of post-sensory nociceptive-sympathetic coupling [1]. In this review, based on the achievements in neurobiology of pain up to now, it is attempted to delineate the underlying neural mechanisms for this post-sensory nociceptive-sympathetic coupling of pain sensation in vertebrates.

\section{Method}

To deal with such a big, important and complex topic, there is no better and more convincing way than integrative reviewing all relevant fields of studies. It is necessary to point out that meta-analysis fits investigation of a specific topic in a well-studied subfield, but not for integrative summarization from several fields of complex anatomic connections. Citing updated relevant reviews or, if not available, salient and repeated experimental results in subfields are the best method.

Papers were searched out from Pubmed and Baidu Xueshu, with such keywords as spinal pain, chronic pain, sympathetic pain, periaqueductal grey, parabrachial area, frog pain, fish pain, and so on. The updated relevant reviews in subfields were given priority to cite. If not available, relevant reviews were cited. If still unavailable, the salient and repeated experimental results of original articles in subfields were cited.

\section{Summarization of Contemporary Nociceptive Feedback Circuits}

\subsection{The Noxious Afferents to the Spinal Cord}

Pain is one of several sensations occurring following stimulation of skin or subcutaneous tissues. Pain results from the afferent activity of nociceptive system devoted to transmission and processing of noxious signals threatening the integrity of the body [2] [3] [4].

All sensory afferents are relayed first via the dorsal root (or posterior root) to the spinal cord with the primary afferent neurons located in dorsal root ganglion (DRG) [2] [3] [4].

There are at least three types of sensory afferent fibers in the peripheral nervous system, the $A ß$-fibers, $A \delta$-fibers, and $\mathrm{C}$-fibers, each of which responds to and relays different types of sensory information [2] [3] [4]. Aß-fibers are large in diameter and highly myelinated, low in activation thresholds and responsible 
for conveying the tactile information [2] [3] [4]. A $\delta$-fibers are smaller in diameter and thinly myelinated, higher in activation thresholds and responsible for both thermal and mechanical information [2] [3] [4]. C-fibers are the smallest type of primary afferents and are unmyelinated, highest in thresholds for activation and responsible for detecting the specific "painful" nociceptive stimuli [2] [3] [4]. Both A $\delta$ - and C-fibers possess nociceptors [3] [4], and can respond to noxious stimuli of mechanical, thermal, and chemical characteristics.

Most of the $\mathrm{A} \delta$-fibers synapse onto the laminae I, II and V of dorsal horn in spinal cord [4] [5], while the C-fibers onto the laminae I and II [4] [5]. In lamina $\mathrm{V}$, there is a type of neurons, termed wide dynamic range (WDR) neurons, which receives input from all Aß-fibers, $\mathrm{A} \delta$-fibers, and C-fibers [4]. All of the neurons in laminae I, II and V in spinal dorsal horn relay nociceptive information (Figure 1).

\subsection{The Periaqueductal Grey and Raphe Feedback}

Many nociceptive projection neurons are located in lamina I of spinal dorsal horn, and send axons to various parts of brain, including the caudal ventrolateral medulla (CVLM), periaqueductal grey (PAG), parabrachial area $(\mathrm{PB})$ and thalamus [4] [5] [6]. The wide dynamic range (WDR) neurons in lamina $\mathrm{V}$ of spinal dorsal horn join the spinothalamic tract [4] [6], and relay the spatially specific pain perception [3].

The PAG in midbrain participates in the endogenous brain inhibition of spinal nociceptive transmission. Activation of PAG neurons excites neurons of the

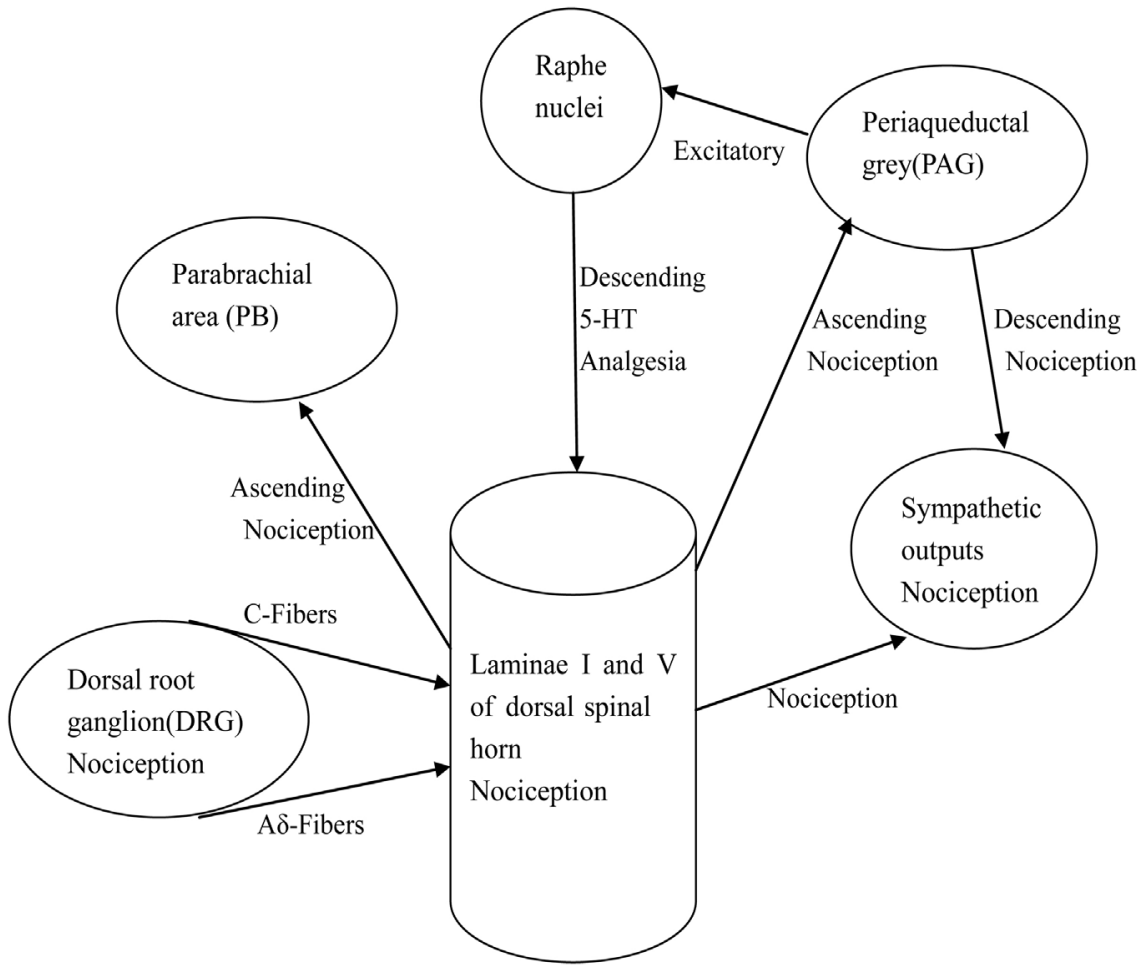

Figure 1. The nociceptive-sympathetic coupling for pain sensation. 
rostral medullary raphe nuclei [7] [8], some of which contain serotonin (5-HT). The 5-HT neurons, in turn, project to the spinal nociceptive neurons and inhibit the firing of them, feedback suppressing their transmission of pain [4] [7] [8].

Collectively, here concisely depict the neural feedback circuits for nociceptive sensation below thalamus that many authors have reached consensus contemporarily [4] [7] [8]: Both A $\delta$ - and C-fibers relay nociceptive sensation to the spinal dorsal horn. Then the spinal nociceptive projection neurons send axons to the PAG, PB and thalamus in brain. Finally, the PAG neurons activate the medullary raphe nuclei which in turn send 5-HT fibers down to inhibit the spinal nociceptive neurons and their transmission of pain (Figure 1).

\section{Addition of Sympathetic Effects of Pain to the Contemporary Nociceptive Circuits}

\subsection{The Sympathetic Effects of Pain}

On May 6, 2016, in television many US athletes of NBA suggested that the sensation of pain might require the postsensory nociceptive-sympathetic coupling [1]. Witnessing the television, Cai wrote a short highlight to briefly introduce their innovative hypothesis [1].

Many evidences can support this hypothesis of NBA. 1) Both causalgia and reflex sympathetic dystrophy manifest as sympathetically maintained pain [9] [10]. 2) The sprouting of sympathetic fibers into the sensory DRG after peripheral nerve injury supports the sympathetic involvement in pain sensation [11] [12]. 3) Sometimes the activation of cardiac/sympathetic reflex and the cardiac parasympathetic deactivation are observed following somatic stimuli during anesthetic surgery [13], demonstrating the presence of subconscious sympathetic/parasympathetic regulation of pain. 4) The sensors and receptors mediating the nociception and pruritus are differentiated but somewhat overlapping with each other [14] [15], demonstrating the requirement of postsensory nociceptive-sympathetic coupling for nociception.

\subsection{The Link of PAG to the Sympathetic Effects of Pain}

Many observations and results demonstrate that the activity of PAG in midbrain links to the associated sympathetic effects of pain. 1) It has just been reported that the muscular sympathetic nerve activity is coupled to the changes in brain activity notably that of PAG during sustained muscle pain [16]. 2) It has also recently been shown that the enhancement in tonic glutamatergic inputs from PAG to rostral ventrolateral medulla (RVLM) contributes to the neuropathic pain-induced high blood pressure [17]. 3) It has been demonstrated that the cardiac sympathetic activation eliciting pain can activate the PAG, PB, dorsal raphe, and locus coeruleus (LC) in brain [18].

Obviously, activation of PAG links to the associated sympathetic effects of pain, completing the post-sensory nociceptive-sympathetic coupling (Figure 1), even though PAG activation concurrently feedbacks to inhibit the spinal noci- 
ceptive transmission via the raphe nuclei.

\subsection{The Direct Link of Spinal Lamina I and V to the Sympathetic Effects of Pain}

Herein it is further added the widely neglected direct sympathetic link of pain via the neurons in lamina I of spinal dorsal horn receiving the nociceptive $\mathrm{A} \delta$ and $\mathrm{C}$-afferents as well as in lamina $\mathrm{V}$ receiving the $\mathrm{A} \delta$-afferents. It was demonstrated that the sympathetic preganglionic neurons received afferents directly from the nociceptive lamina I [19] and V [20] in spinal dorsal horn. Besides, it was further demonstrated that the sympathetic preganglionic neurons could be activated by these nociceptive neurons in spinal dorsal horn [21] [22] (Figure 1).

Obviously, activation of the sympathetic preganglionic neurons by lamina I and $\mathrm{V}$ in spinal dorsal horn directly links to the associated sympathetic effects of pain, substantiating the post-sensory nociceptive-sympathetic coupling, even though such link has seldom been studied up to now and is required more investigations in future.

\section{The Primitive Pain Coupling in Evolution of Vertebrates}

\subsection{The Link of PB to the Respiratory Effects of Pain}

Noxious stimulation causing cutaneous nociception can elicit increase in breathing [23]. The nociceptive neurons in lamina I of spinal dorsal horn also send axons to the CVLM, PB and thalamus in addition to PAG [4] [5] [6]. It was reported that the pontine $\mathrm{PB}$ neurons contributed to the cutaneous nociceptor-induced increase in breathing [23]. Thus, corresponding to the link of PAG and spinal cord to the sympathetic effects of pain, the PB links to the associated respiratory effects of pain.

\subsection{The Primitive Pain Coupling in Evolution of Vertebrates}

Because the PAG, PB and raphe nuclei are all located in brain below thalamus, they are evolutionarily ancient and primitive in vertebrates. The PAG and spinal cord link to the associated sympathetic effects of pain, the PB to the respiratory effects, while the raphe 5-HT feedbacks to inhibit the spinal nociceptive transmission (Figure 1). Accordingly, the sympathetic/respiratory effects of pain and the 5-HT inhibition of spinal nociceptive transmission are all below thalamus and primitive in vertebrate evolution.

Progressions of pain research in fishes reveal that pain really exists in fishes [24] [25] [26]. On the one hand, it has been demonstrated that the teleost fish possesses nociceptors innervated by the trigeminal nerve which are physiologically similar to those found in higher vertebrates [24] [25], demonstrating the involvement of brain in pain perception early in fish. Opioid receptors and endogenous opioids are found in the fish brain and spinal cord while morphine reduces the behavioral and physiological nociceptive responses to noxious stimulation [24] [25]. On the other hand, it has been demonstrated that the neu- 
ronal responses can be elicited in spinal cord, cerebellum, tectum, and telencephalon in both goldfish and trout during brush (mechanoceptive) and pin-prod (nociceptive) stimuli [26], demonstrating the involvement of both brain and spinal cord in pain perception in fish.

However, there is only gill rather than lung in fish, and accordingly the nociceptive effects of pontine PB neurons to breathing [23] may be different. The lung appeared after amphibians having emerged in evolution. It has been demonstrated that the nociceptors [27] as well as the $\mathrm{A} \delta$ - and C-fibers [27] [28] are present in frogs. Anatomically, these amphibian central nociceptive circuits are mainly related to spinal cord and their long loop reflexes to the midbrain and thalamus [27].

Obviously, the nociceptive functions of midbrain and spinal cord were acquired early in vertebrate evolution even in fishes and amphibians. Because the PAG, PB and raphe nuclei are all located in the primitive brain below thalamus and ancient in vertebrate evolution, the relevant sympathetic effects of pain and the 5-HT feedback inhibition of spinal nociceptive transmission would speculatively both be ancient in evolution early in fishes and amphibians, which requires more investigations.

\section{Discussions}

In this review, based on the proposal of US athletes of NBA in television that the pain sensation might result necessarily from the postsensory nociceptive-sympathetic coupling mechanism, it is attempted to delineate the related neural pathways to support this nociceptive-sympathetic coupling of pain sensation in vertebrates.

In the contemporary feedback neural circuits of pain transmission in spinal cord, the afferent $\mathrm{A} \delta$-fibers relay nociceptive sensations to the laminae I, II and V of spinal dorsal horn [2] [3] [4] [5], and the C-fibers to the laminae I, II there [2] [3] [4] [5]. The spinal nociceptive neurons in these laminae send axons to the PAG, PB and thalamus in brain [4] [5] [6]. As feedback, the PAG neurons activate the medullary raphe nuclei which in turn send 5-HT fibers down to inhibit the spinal nociceptive transmission [4] [7] [8].

Such feedback circuits of pain transmission do not consider the sympathetic effects of pain, certainly not competent to delineate the neural pathways underlying the post-sensory nociceptive-sympathetic coupling. In this article, it is extended to cite the nociceptive neurons in laminae I and $\mathrm{V}$ of dorsal spinal horn [19] [20] [21] [22], as well as those in PAG [16] [17] [18], to additionally activate the sympathetic outputs, completing the post-sensory nociceptive-sympathetic coupling. Correspondingly, the PB neurons associate to the post-sensory respiratory effects of pain [23].

It could be argued that the nociceptive-sympathetic coupling contributes only to the sympathetic output from pain rather than the sensation of pain. However, all sympathetic reactions have central feelings. The sympathetic sensation from 
pain must be coherent with the sensation of pain. In this regard, the nociceptive-sympathetic coupling would contribute to a portion of pain sensation. As evidence, both causalgia and reflex sympathetic dystrophy manifest as sympathetically maintained pain [9] [10]. Besides, the sprouting of sympathetic fibers into the sensory DRG after peripheral nerve injury further supports the sympathetic involvement in pain sensation [11] [12].

It is noted that some brain structures above thalamus are also involved in pain perception, such as the thalamus and cortex [3], which may also contribute to the post-sensory nociceptive-sympathetic coupling. However, these higher brain structures are gradually acquired during vertebrate evolution. The PAG, PB, raphe nuclei and spinal cord adopted in this article are all primitive below thalamus and ancient in vertebrate evolution. The demonstrations of pain in fishes and frogs reveal that both midbrain and spinal cord have acquired the nociceptive functions in these ancient vertebrates [24] [25] [26] [27] [28]. In this regard, it is plausible to apply the neural pathways of post-sensory nociceptive-sympathetic coupling here even to the fishes and amphibians, and to make it universal to most vertebrate species.

\section{Limitations}

Even though this review supports the new post-sensory nociceptive-sympathetic coupling hypothesis of NBA for pain sensation, it only considers the primitive brain structures below thalamus of pain which are conserved in vertebrate evolution. The brain structures above thalamus, such as the thalamus and cortex [3], are also involved in pain perception. Besides, beyond the nociceptive-sympathetic coupling, there may exist other additional mechanisms for pain sensation, such as cerebral neuronal plasticity as well for chronic pain [29], and so on.

\section{Conclusion}

In this review, it is delineated the underlying neural pathways to support for the hypothetic post-sensory nociceptive-sympathetic coupling of NBA for pain sensation. The nociceptive neurons in laminae I and V of dorsal spinal horn as well as those in PAG can additionally activate the sympathetic outputs, so that they complete the post-sensory nociceptive-sympathetic coupling. These nociceptive-sympathetic coupling pathways would definitely contribute to a portion of pain sensation because of sympathetically maintained pain. Likewise, the PB associates with the post-sensory respiratory effects of pain. Because both PAG and $\mathrm{PB}$ are below thalamus and primitive, these sympathetic and respiratory associations in pain by both midbrain and spinal cord must be primitive and universal in vertebrate evolution, even plausibly acquired early in fishes and amphibians for the sympathetic effects while in amphibians for the respiratory effects of pain.

\section{Acknowledgements}

It is herein acknowledged that Mingxun Cai paid the Open Access publication 
fee of this paper.

\section{Funding}

The author declares no financial support for this work.

\section{Conflicts of Interest}

The author declares no conflict of interest or financial support for this work.

\section{References}

[1] Cai, Z.-J. (2016) The Hypothetic Post-Sensory Nociceptive-Sympathetic Coupling Necessary for Pain Sensation from NBA. Journal of Neurology and Neuroscience, 7, 141. https://doi.org/10.21767/2171-6625.1000141

[2] Lawson, S.N. (2002) Phenotype and Function of Somatic Primary Afferent Nociceptive Neurones with C-, Adelta- or Aalpha/Beta-Fibres. Experimental Physiology, 87, 239-244. https://doi.org/10.1113/eph8702350

[3] Haggard, P., Iannetti, G.D. and Longo, M.R. (2013) Spatial Sensory Organization and Body Representation in Pain Perception. Current Biology, 23, R164-R176. https://doi.org/10.1016/j.cub.2013.01.047

[4] D’Mello, R. and Dickenson, A.H. (2008) Spinal Cord Mechanisms of Pain. British Journal of Anaesthesia, 101, 8-16. https://doi.org/10.1093/bja/aen088

[5] Todd, A.J. (2002) Anatomy of Primary Afferents and Projection Neurones in the Rat Spinal Dorsal Horn with Particular Emphasis on Substance P and the Neurokinin 1 Receptor. Experimental Physiology, 87, 245-249. https://doi.org/10.1113/eph8702351

[6] Kobayashi, Y. (1998) Distribution and Morphology of Spinothalamic Tract Neurons in the Rat. Anatomy and Embryology, 197, 51-67. https://doi.org/10.1007/s004290050119

[7] Basbaum, A.I. and Fields, H.L. (1978) Endogenous Pain Control Mechanisms: Review and Hypothesis. Annals of Neurology, 4, 451-462. https://doi.org/10.1002/ana.410040511

[8] Mayer, D.J. (1984) Analgesia Produced by Electrical Stimulation of the Brain. Progress in Neuro-Psychopharmacology \& Biological Psychiatry, 8, 557-564. https://doi.org/10.1016/0278-5846(84)90015-0

[9] Roberts, W.J. (1986) A Hypothesis on the Physiological Basis for Causalgia and Related Pains. Pain, 24, 297-311. https://doi.org/10.1016/0304-3959(86)90116-8

[10] Gibbs, G.F., Drummond, P.D., Finch, P.M. and Phillips, J.K. (2008) Unravelling the Pathophysiology of Complex Regional Painsyndrome: Focus on Sympathetically Maintained Pain. Clinical and Experimental Pharmacology and Physiology, 35, 717-724. https://doi.org/10.1111/j.1440-1681.2007.04862.x

[11] Chen, S.S. and Zhang, J.M. (2015) Progress in Sympathetically Mediated Pathological Pain. Journal of Anesthesia and Perioperative Medicine, 2, 216-225. https://doi.org/10.24015/JAPM.2015.0029

[12] Xie, W., Strong, J.A., Li, H. and Zhang, J.M. (2007) Sympathetic Sprouting Near Sensory Neurons after Nerve Injury Occurs Preferentially on Spontaneously Active Cells and Is Reduced by Early Nerve Block. Journal of Neurophysiology, 97, 492-502. https://doi.org/10.1152/jn.00899.2006

[13] Cividjian, A., Petitjeans, F., Liu, N., Ghignone, M., de Kock, M. and Quintin, L. 
(2017) Do We Feel Pain during Anesthesia? A Critical Review on Surgery-Evoked Circulatory Changes and Pain Perception. Best Practice \& Research Clinical Anaesthesiology, 31, 445-467. https://doi.org/10.1016/j.bpa.2017.05.001

[14] Liu, T. and Ji, R.R. (2013) New Insights into the Mechanisms of Itch: Are Pain and Itch Controlled by Distinct Mechanisms? Pflügers Archiv-European Journal of Physiology, 465, 1671-1685. https://doi.org/10.1007/s00424-013-1284-2

[15] Baraniuk, J.N. (2012) Rise of the Sensors: Nociception and Pruritus. Current Allergy and Asthma Reports, 12, 104-114. https://doi.org/10.1007/s11882-012-0245-8

[16] Kobuch, S., Fazalbhoy, A., Brown, R., Macefield, V.G. and Henderson, L.A. (2018) Muscle Sympathetic Nerve Activity-Coupled Changes in Brain Activity During Sustained Muscle Pain. Brain and Behavior, 8, e00888.

https://doi.org/10.1002/brb3.888

[17] Wang, W., Zou, Z., Tan, X., Zhang, R.W., Ren, C.Z., Yao, X.Y., Li, C.B., Wang, W.Z. and Shi, X.Y. (2017) Enhancement in Tonically Active Glutamatergic Inputs to the Rostral Ventrolateral Medulla Contributes to Neuropathic Pain-Induced High Blood Pressure. Neural Plasticity, 2017, Article ID: 4174010.

https://doi.org/10.1155/2017/4174010

[18] Guo, Z., Li, P. and Longhurst, J.C. (2002) Central Pathways in the Pons and Midbrain Involved in Cardiac Sympathoexcitatory Reflexes in Cats. Neuroscience, 113, 435-447. https://doi.org/10.1016/S0306-4522(02)00173-2

[19] Craig, A.D. (1993) Propriospinal Input to Thoracolumbar Sympathetic Nuclei from Cervical and Lumbar Lamina I Neurons in the Cat and the Monkey. Journal of Comparative Neurology, 331, 517-530. https://doi.org/10.1002/cne.903310407

[20] Cabot, J.B., Alessi, V., Carroll, J. and Ligorio, M.(1994) Spinal Cord Lamina V and Lamina VII Interneuronal Projections to Sympathetic Preganglionic Neurons. Journal of Comparative Neurology, 347, 515-530. https://doi.org/10.1002/cne.903470404

[21] Dembowsky, K., Czachurski, J. and Seller, H. (1985) An Intracellular Study of the Synaptic Input to Sympathetic Preganglionic Neurones of the Third Thoracic Segment of the Cat. Journal of the Autonomic Nervous System, 13, 201-244.

https://doi.org/10.1016/0165-1838(85)90012-8

[22] Spanswick, D., Renaud, L.P. and Logan, S.D. (1998) Bilaterally Evoked Monosynaptic EPSPs, NMDA Receptors and Potentiation in Rat Sympathetic Preganglionic Neurones in Vitro. The Journal of Physiology, 509, 195-209. https://doi.org/10.1111/j.1469-7793.1998.195bo.x

[23] Jiang, M., Alheid, G.F., Calandriello, T. and McCrimmon, D.R. (2004) Parabrachial-Lateral Pontine Neurons Link Nociception and Breathing. Respiratory Physiology \& Neurobiology, 143, 215-233. https://doi.org/10.1016/j.resp.2004.07.019

[24] Sneddon, L.U. (2015) Pain in Aquatic Animals. Journal of Experimental Biology, 218, 967-976. https://doi.org/10.1242/jeb.088823

[25] Sneddon, L.U. (2004) Evolution of Nociception in Vertebrates: Comparative Analysis of Lower Vertebrates. Brain Research Reviews, 46, 123-130. https://doi.org/10.1016/j.brainresrev.2004.07.007

[26] Dunlop, R. and Laming, P. (2005) Mechanoreceptive and Nociceptive Responses in the Central Nervous System of Goldfish (Carassius auratus) and Trout (Oncorhynchus mykiss). The Journal of Pain, 6, 561-568. https://doi.org/10.1016/j.jpain.2005.02.010

[27] Guénette, S.A., Giroux, M.C. and Vachon, P. (2013) Pain Perception and Anaesthesia in Research Frogs. Experimental Animals, 62, 87-92. 
[28] Hamamoto, D.T. and Simone, D.A. (2003) Characterization of Cutaneous Primary Afferent Fibers Excited by Acetic Acid in a Model of Nociception in Frogs. Journal of Neurophysiology, 90, 566-577. https://doi.org/10.1152/jn.00324.2003

[29] Zhuo, M. (2016) Contribution of Synaptic Plasticity in the Insular Cortex to Chronic Pain. Neuroscience, 338, 220-229.

https://doi.org/10.1016/j.neuroscience.2016.08.014 